\title{
A Case of Vogt-Koyanagi-Harada Syndrome with Persistent Dyspnea Secondary to Laryngeal Edema
}

\author{
Dimosthenis Mantopoulos $^{a} \quad$ Brad W. deSilva $^{b} \quad$ Colleen M. Cebulla ${ }^{a}$ \\ ${ }^{a}$ Havener Eye Institute, Department of Ophthalmology and Visual Sciences, and \\ ${ }^{b}$ Department of Otolaryngology - Head and Neck Surgery, The Ohio State University \\ Wexner Medical Center, Columbus, Ohio, USA
}

\section{Key Words}

Vogt-Koyanagi-Harada syndrome - Laryngeal edema - Glottic edema - Autoimmune disease · Laryngoscopy

\begin{abstract}
Purpose: We report a case of laryngeal edema associated with the Vogt-Koyanagi-Harada (VKH) syndrome. Patient and Methods: A 32-year-old African-American female presented with a 12-day prodrome, including headache, tinnitus and shortness of breath, which preceded sudden photophobia and bilateral visual loss. Examination and clinical testing were most consistent with $\mathrm{VKH}$, and the patient improved with intravenous methylprednisolone therapy. Results: The patient had persistent dyspnea, which was out of proportion to chest CT findings and which was exacerbated during a recurrence of VKH. Flexible fiberoptic laryngoscopy with stroboscopy revealed diffuse laryngeal edema. Symptoms were alleviated with breathing exercises. Conclusions: Several autoimmune diseases may cause diffuse laryngeal edema. In this case, VKH was associated with the patient's glottic edema and dyspnea. We recommend that laryngeal edema be considered in the differential diagnosis for patients with dyspnea and VKH.

(c) 2014 S. Karger AG, Basel
\end{abstract}

\section{Introduction}

The Vogt-Koyanagi-Harada (VKH) syndrome is an autoimmune systemic disease of unknown etiology characterized by recurrent episodes of non-necrotizing bilateral panuveitis. The granulomatous inflammation affects different tissues in the body, the common characteristic of which is the presence of melanocytes [1]. Thus, patients have 
Mantopoulos et al:: A Case of Vogt-Koyanagi-Harada Syndrome with Persistent Dyspnea Secondary to Laryngeal Edema

various manifestations secondary to eye, ear, skin and meningeal involvement. Here, we present a unique case of VKH with dyspnea, secondary to laryngeal edema.

\section{Patient and Methods}

The patient was a healthy 32-year-old African-American woman employed as a loan specialist. She had a 12-day history of headache, tinnitus, earache, non-productive cough, rhinorrhea, epistaxis and dyspnea together with constitutional symptoms of night sweats and weight loss. She then woke up with blurry vision and photophobia accompanied by conjunctival injection for 1-2 days. The visual acuity measured was 20/300 OD and 20/200 OS. Both eyes had posterior synechiae, keratic precipitates, anterior chamber cell and flare, and trace vitreous cells. Dilated fundus examination revealed disc hyperemia, macular edema and bilateral multifocal serous retinal detachments (RDs) OU. Fluorescein angiography was significant for hyperfluorescence of the optic nerve (OS > OD) and small hyperfluorescent spots with leakage in detached parts of the macula OS. Optical coherence tomography revealed serous RDs with subretinal fluid (OU). Testing to measure choroidal thickness with enhanced depth imaging optical coherence tomography or ultrasound was not performed.

Laboratory testing was within normal limits, including rapid plasma reagin, the cerebrospinal fluid (CSF) venereal disease research laboratory test, CSF and peripheral blood angiotensin-converting enzyme, CSF analysis (gross appearance, cell count, protein, glucose), blood cultures, toxoplasmosis PCR, the QuantiFERON-TB test, double-stranded DNA, lupus anticoagulant, antinuclear antibody, rheumatoid factor, cryoglobulin/cryofibrinogen, complement C3/C4, SS-A and SS-B antibodies, anti-Smith antibody, ribonuclear protein antibody, $\beta_{2}$-glycoprotein, anticardiolipin antibody, hepatitis $\mathrm{C}$ antibody, anticentromere, antineutrophil cytoplasmic antibody, antimyeloproxidase, and anti-Jo-1 antibody. Lysozyme was elevated on first presentation and then normalized. This case report complied with the guidelines for human studies, and the patient has given informed consent for publication.

\section{Results}

Magnetic resonance imaging and angiography of the brain showed no evidence of vasculitis or other abnormalities. The chest CT was clear except for mild nonspecific changes including right lower lobe atelectasis and scattered, small noncalcified nodules including a 4mm lingular nodule and a 3-mm right upper lobe nodule.

At that time, given the ocular, neurologic and auditory manifestations as well as the absence of ocular trauma and other ocular diseases, the patient met 4 out of 5 of the modified diagnostic criteria of the VKH syndrome [2]. The patient's ocular inflammation improved significantly with a 3-day course of intravenous methylprednisolone $1 \mathrm{~g}$ daily, so the patient was discharged on a tapering dose of prednisone with resolution of serous RDs by 1.5 weeks after treatment. However, tapering or replacing the prednisone with steroidsparing azathioprine, beginning 7 weeks after initial treatment, as well as a 3-day gap without prednisone at week 9, led to the loss of visual acuity, including a new episode of acute bilateral vision loss. Recurrent uveitis with subretinal fluid OU was identified (fig. 1c, d), and the patient was treated with a bilateral sub-Tenon's triamcinolone ( $40 \mathrm{mg}$ ) injection and pulsed high-dose intravenous methylprednisolone. The ocular symptoms were 
Mantopoulos et al.: A Case of Vogt-Koyanagi-Harada Syndrome with Persistent Dyspnea Secondary to Laryngeal Edema

accompanied by tinnitus and marked dyspnea. Pulmonary consultation identified nonspecific pulmonary findings which were out of proportion to her dyspnea. Pulmonary function tests showed mild restrictive disease and mild nonspecific findings. Otolaryngology was consulted, and the flexible fiberoptic laryngoscopy with stroboscopy revealed complete glottic closure, moderate edema of both true and false vocal folds, aryepiglottic folds, and postcricoid mucosa (fig. 2). Vocal cord exercises in the clinic alleviated her dyspnea symptoms. Her ocular disease was controlled with methotrexate and slow steroid tapering, with a resolution of RDs by 8 months after initial presentation.

\section{Discussion}

To the best of our knowledge, this is the first case of VKH syndrome and persistent dyspnea secondary to laryngeal edema reported in the literature. The mechanism of the patient's dyspnea, which was out of proportion to her mild nonspecific pulmonary changes, was glottic edema caused by a narrowed laryngeal inlet, leading to dyspnea symptoms that are more prominent on inspiration but can be biphasic. Some common causes for diffuse laryngeal edema include obstructive sleep apnea, laryngeal sicca, hypothyroidism, laryngopharyngeal reflux, allergic laryngitis, and autoimmune or inflammatory processes such as Sjögren's syndrome, sarcoidosis, hereditary angioedema, and pemphigoid [3, 4]. Melanocytes can be found in the larynx [5] and could have been the target of the autoimmune process. We suspect that in this case, VKH was the underlying inflammatory condition responsible for the patient's laryngeal edema.

\section{Acknowledgments}

Supported by the National Eye Institute of the National Institutes of Health under Award Number K08EY022672. Additional funds were provided by the Ohio Lions Eye Research Foundation, Fund No. 313310 in Ophthalmology, and the Patti Blow Fund.

\section{Disclosure Statement}

The authors declare no conflicts of interest.

\section{References}

1 Greco A, Fusconi M, Gallo A, Turchetta R, Marinelli C, et al: Vogt-Koyanagi-Harada syndrome. Autoimmun Rev 2013;12:1033-1038.

-2 Read RW, Holland GN, Rao NA, Tabbara KF, Ohno S, et al: Revised diagnostic criteria for Vogt-KoyanagiHarada disease: report of an international committee on nomenclature. Am J Ophthalmol 2001;131:647652.

3 Abdel-Aziz M, Azab NA, Bassyouni IH, Hamdy G: Laryngeal involvement in juvenile idiopathic arthritis patients. Clin Rheumatol 2011;30:1251-1256.

4 Chiang T, Marcinow AM, deSilva BW, Ence BN, Lindsey SE, et al: Exercise-induced paradoxical vocal fold motion disorder: diagnosis and management. Laryngoscope 2013;123:727-731.

5 Goldman JL, Lawson W, Zak FG, Roffman JD: The presence of melanocytes in the human larynx. Laryngoscope 1972;82:824-835. 
Case Reports in

Ophthalmology
Case Rep Ophthalmol 2014;5:361-364

DOI: $10.1159 / 000369003$

Mantopoulos et al.: A Case of Vogt-Koyanagi-Harada Syndrome with Persistent Dyspnea Secondary to Laryngeal Edema
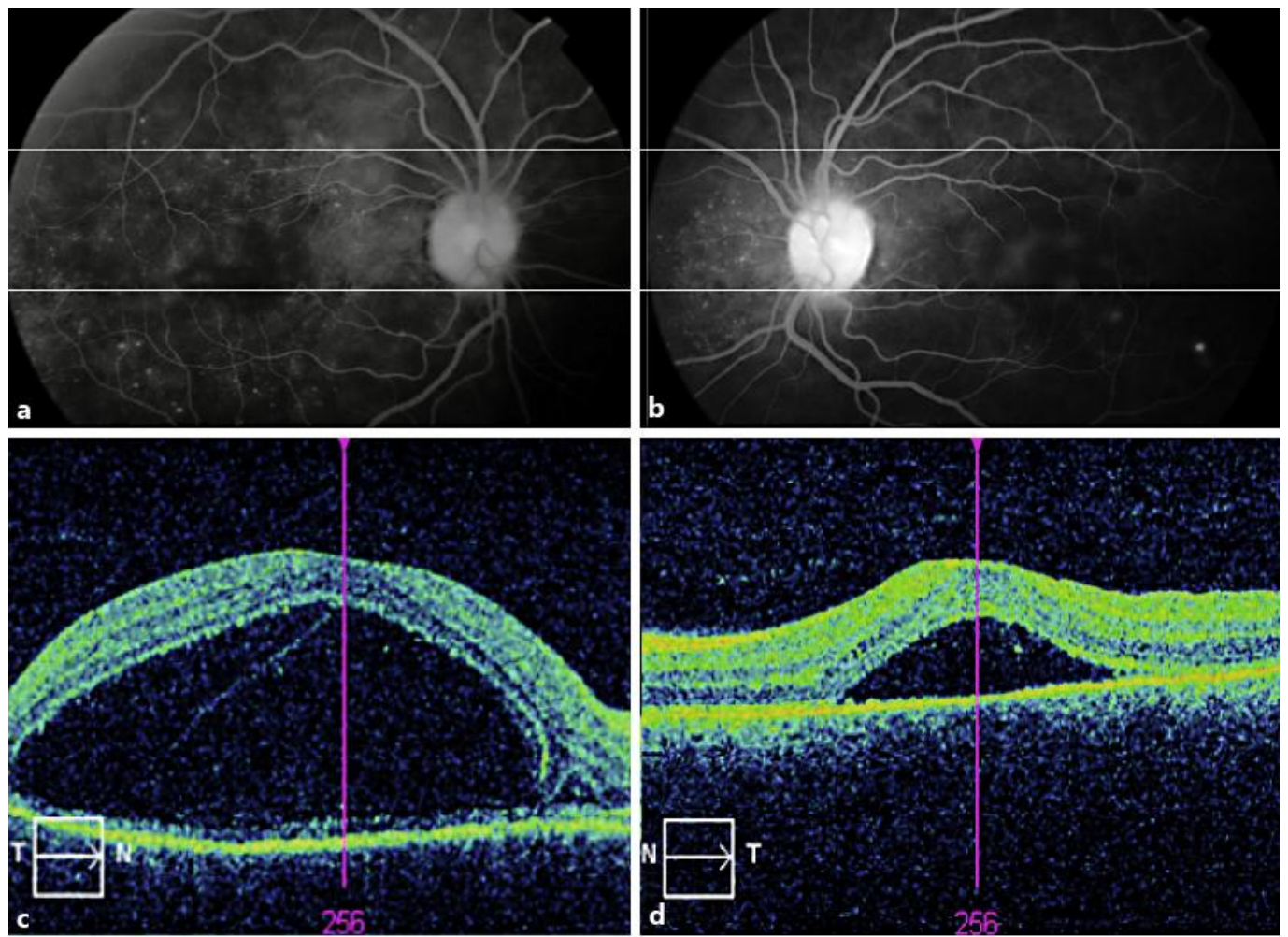

Fig. 1. Late-phase fluorescein angiography of both eyes significant for multiple areas of hyperfluorescence with late leakage and optic disc staining $(\mathbf{a}, \mathbf{b})$. Spectral domain optical coherence tomography of the right (c) and left (d) eye shows subretinal fluid and debris on the retinal pigment epithelium.

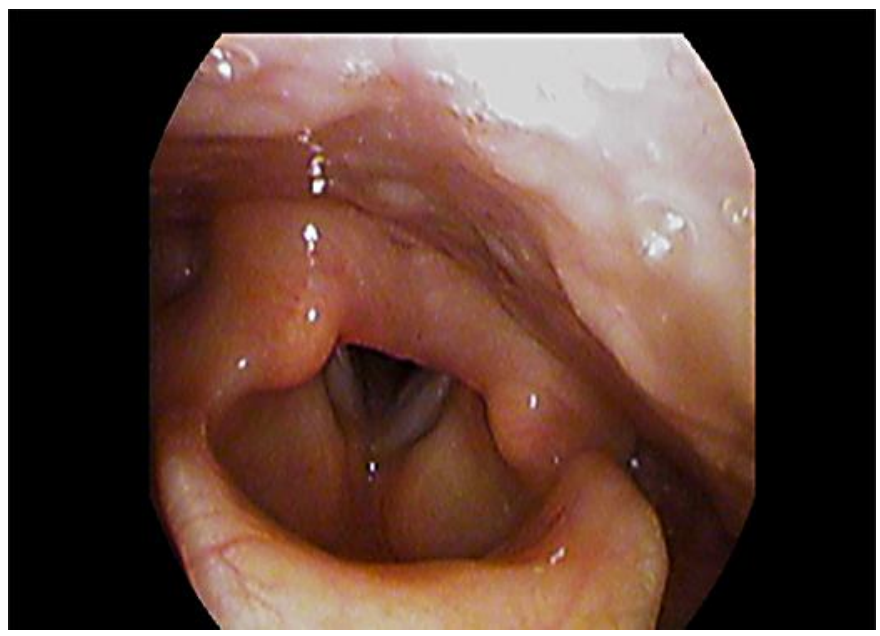

Fig. 2. Laryngoscopic photograph showing diffuse edema of the glottic and supraglottic mucosa. 